\title{
EXAMINATION OF LOPERAMIDE AND CROFELEMER IN A RAT MODEL OF NERATINIB-INDUCED DIARRHEA
}

Joanne Bowen ${ }^{1}$, Kate Secombe ${ }^{1}$, Imogen Ball ${ }^{1}$, Joseph Shirren ${ }^{1}$, Anthony Wignall ${ }^{1}$, Hannah Wardill ${ }^{2}$, Ysabella Van Sebille 3 , Francesca Avogadri-Connors ${ }^{4}$, Susan Moran ${ }^{4}$, Elizabeth Olek ${ }^{4}$, David Martin ${ }^{4}$

(1) Adelaide Medical School, University of Adelaide, Australia, (2) Centre for Nutrition and Gastrointestinal Diseases,

South Australian Health and Medical Research Institute, Australia, (3) Division of Health Sciences, University of South Australia, Australia, (4) Puma Biotechnology, Inc, Los Angeles, USA

\section{Introduction}

\section{Neratinib}

$>$ Irreversible small molecule pan-ErbB tyrosine kinase inhibitor

$>$ Approved for adjuvant treatment of HER2 ${ }^{+}$breast cancer

$>$ Associated with $40 \%$ grade $3+$ diarrhea when no prophylactic antidiarrheal used ${ }^{1}$

Model development

$>$ Our group has established a rat model of neratinib-induced diarrhea to investigate new interventions

- Current standard of care for diarrhea is loperamide

$>$ A novel agent, crofelemer, has been suggested to target mechanisms of diarrhea by preventing epithelial chloride secretion ${ }^{2}$

$>$ May be a new prophylaxis approach

Figure 1: Receptor tyrosine kinase inhibitor mechanism of action for neratinib

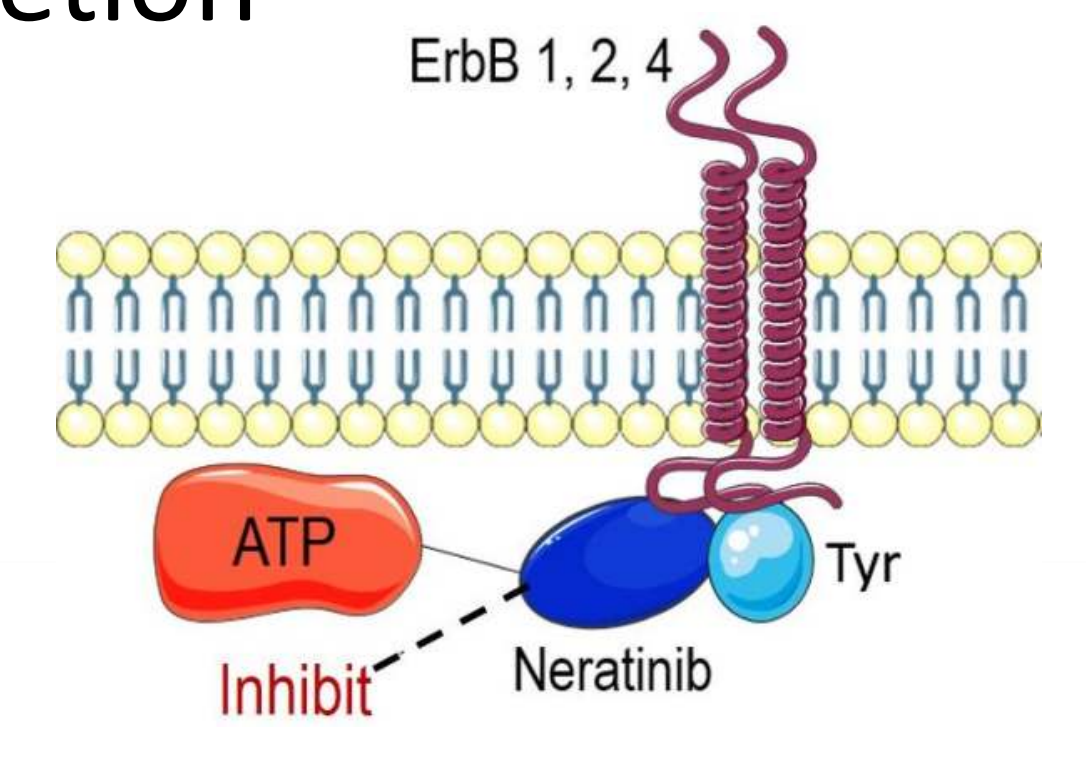

\section{Study Objective}

To compare effectiveness of loperamide vs crofelemer for prevention of neratinib-induced diarrhea in a rat model

\section{Specific aims:}

1) To assess impact on diarrhea incidence and severity

2) To determine effect on neratinib absorption

3) To quantify changes to intestinal physiology \& permeability

\section{Materials and Methods}

\section{Animal model}

64 male albino Wistar rats treated daily by oral gavage with either:

$>50 \mathrm{mg} / \mathrm{kg}$ neratinib

$>50 \mathrm{mg} / \mathrm{kg}$ neratinib $+0.4 \mathrm{mg} / \mathrm{kg}$ loperamide $\mathrm{HCl}$

$>50 \mathrm{mg} / \mathrm{kg}$ neratinib $+25 \mathrm{mg} / \mathrm{kg}$ crofelemer

$>$ vehicle control $(0.5 \% \mathrm{HPMC})$

Groups of rats $(n=4)$ were killed at 7, 14, 21 and 28 days.

\section{Diarrhea and neratinib analysis}

Rats were assessed for weight loss and diarrhea daily using an established grading system ( $0=$ none, $1=$ soft, $2=$ wet, $3=$ wet with extended fur staining). Serum neratinib was measured weekly $\left(\mathrm{C}_{\min }-24 \mathrm{~h}\right.$ after previous dose) or over $24 \mathrm{~h}$ (pharmacokinetic - 0, 1, 3, 6, 9, 12, 24 h) by LC-MS/TOF.

\section{Tissue electrophysiological analysis}

Fresh ileum and colon tissue was stripped of the muscle layer and mounted in Ussing chambers to assess permeability and ion conductance.

\section{Statistical analysis}

Repeated measures 2-way ANOVA and Freidman's test: weight gain, serum neratinib and diarrhea incidence and severity.

One-way ANOVA and Kruskal-Wallis test: Permeability and conductance Data shown as mean +/- SEM unless otherwise stated.

\section{Results \\ 1. No improvement in neratinib-induced diarrhea with loperamide or crofelemer treatment \& more pronounced delay in weight gain}
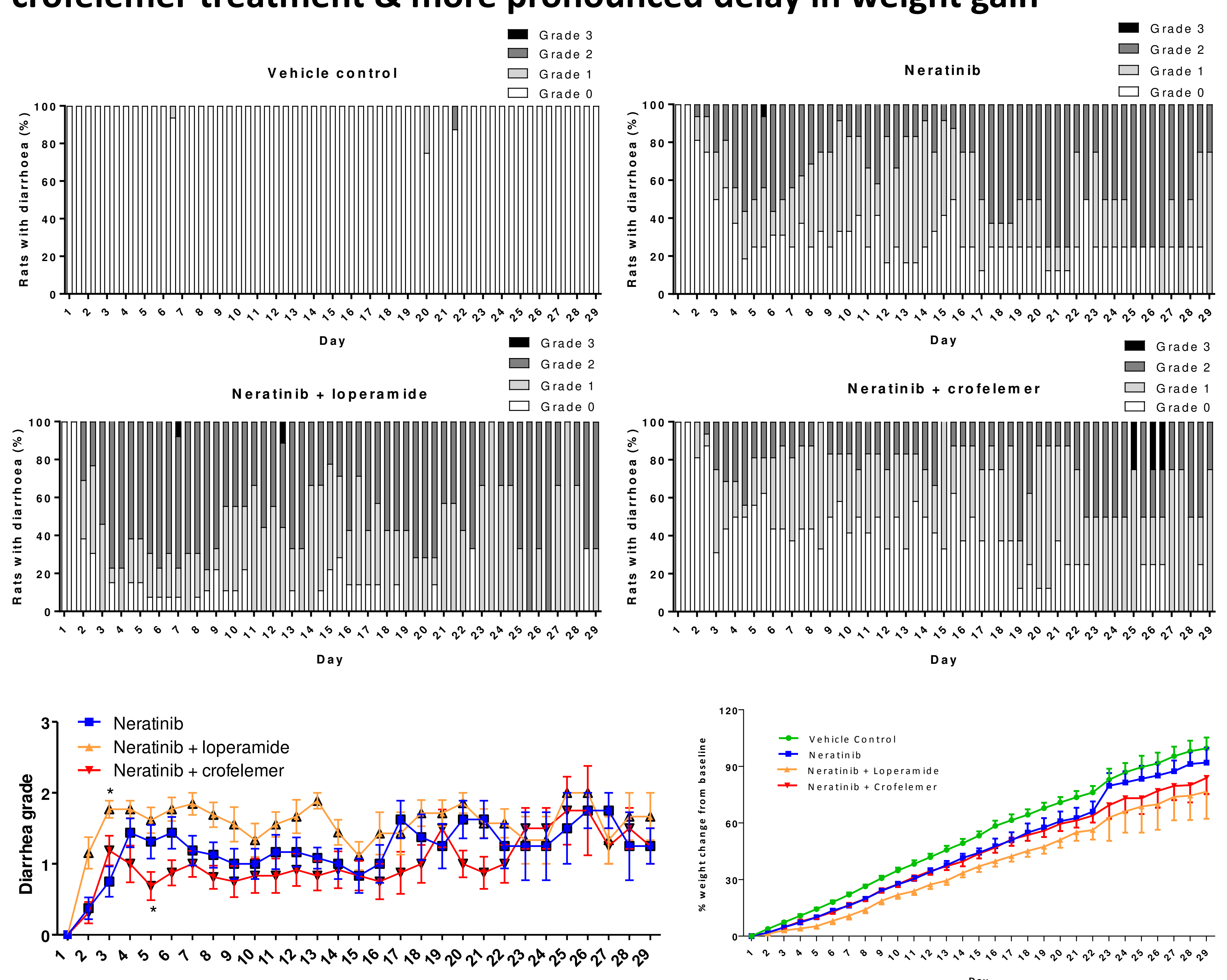

2. Loperamide increased serum neratinib concentration at $\mathbf{2}$ weeks $\&$ crofelemer decreased absorption after a single dose
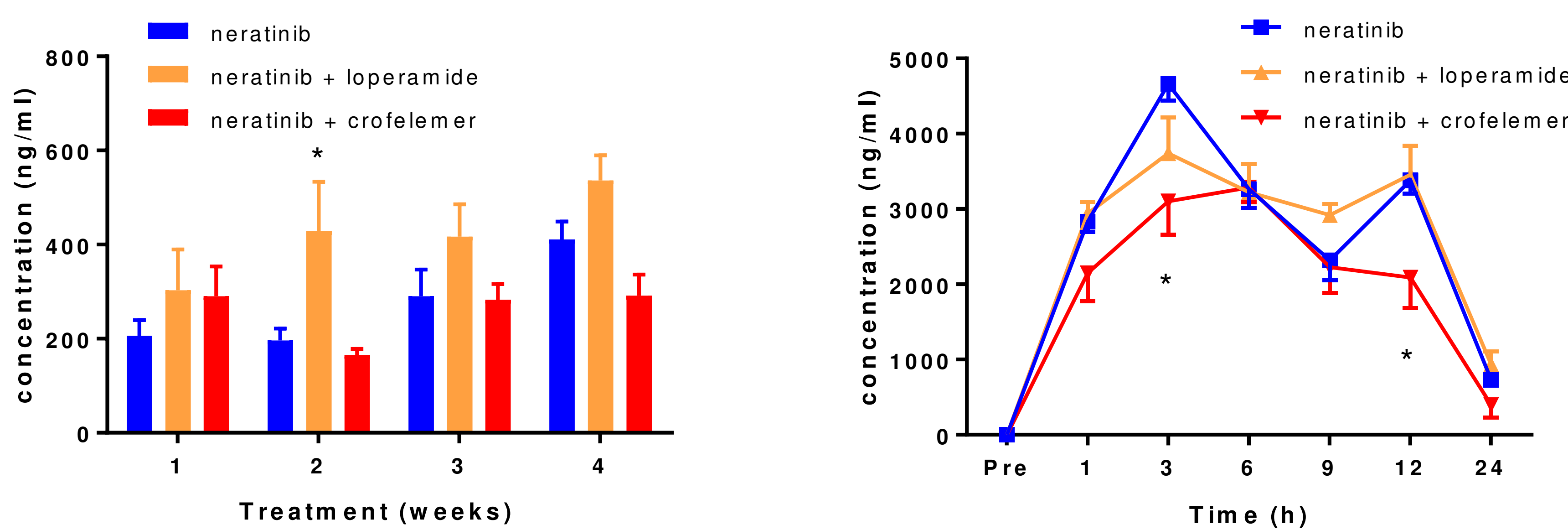

3. Mucosal permeability and ion conductance were not significantly altered by loperamide or crofelemer after 28 days of treatment
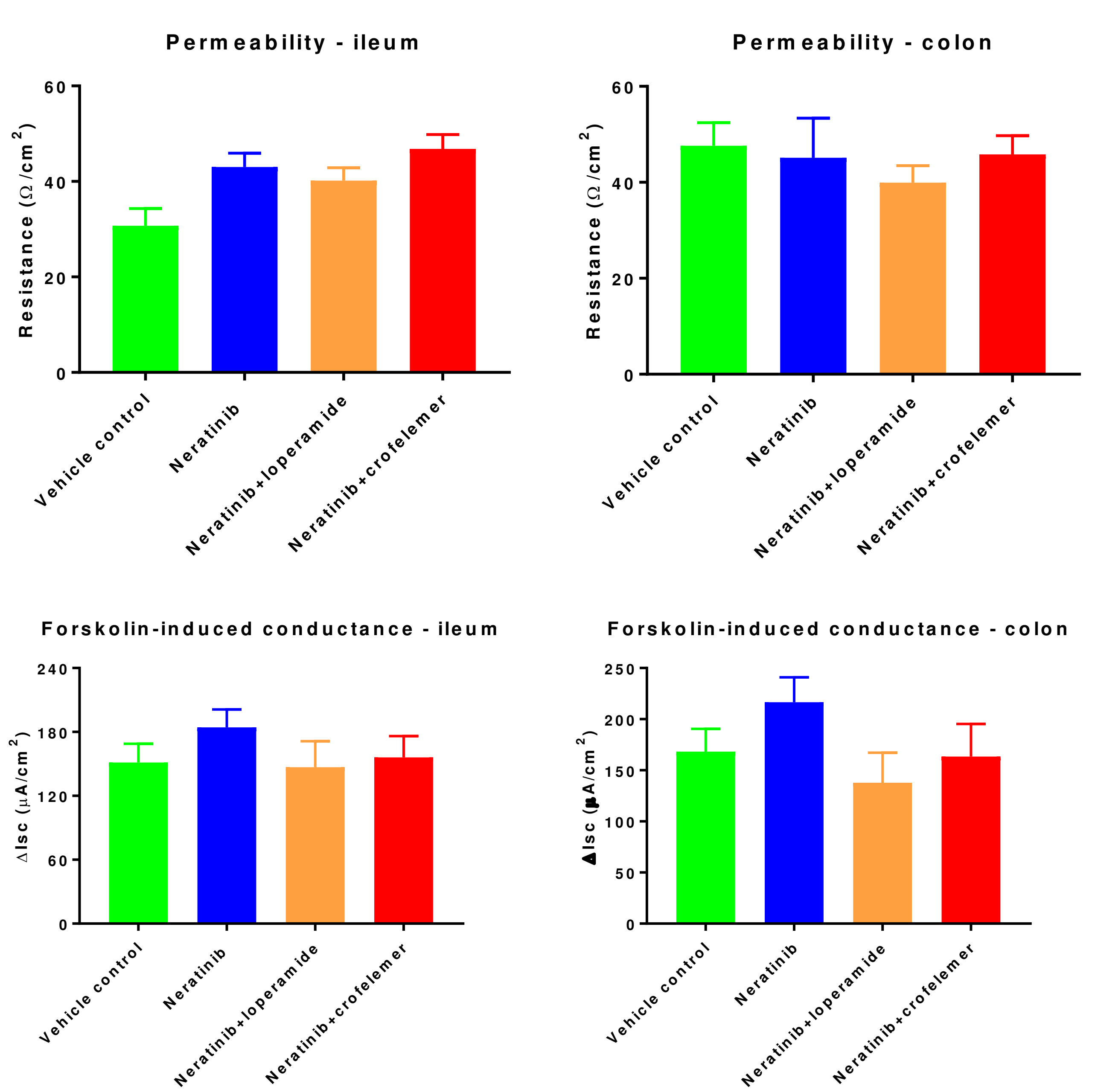

\section{Conclusions}

- This study found no evidence of effectiveness for the anti-diarrheal agents, loperamide and crofelemer, for prevention of neratinib-induced diarrhea.

- Serum neratinib was increased in rats co-treated with loperamide $(303 \mathrm{ng} / \mathrm{ml})$ compared to neratinib alone $(198 \mathrm{ng} / \mathrm{ml})$ on day 14 and may be associated with altered metabolism. This coincided with worse average grade diarrhea compared to neratinib alone and crofelemer-treated rats during the first 14 days. Crofelemer was associated with a decrease in 0-24 h AUC (39890 $\mathrm{ng} * \mathrm{~h} / \mathrm{ml})$ compared to neratinib alone $(60983 \mathrm{ng}$ (h/ml) after a single dose, but did not alter weekly concentrations. This likely reflects that crofelemer is not systemically absorbed.

- Resistance was not significantly changed indicating that tissue injury did not lead to increased permeability. Non-significant changes in ion conductance suggest that neratinib-induced diarrhea is not driven by a chloride secretory mechanism in our rat model and other mechanisms should be explored. 